The accurate interpretation of these ares takes considerable practice. The most important parameter is the position of the peak of the arc. These are indicated by arrows in Fig. 2. The interpretation of the length and shape of the arc will follow with practice if attention is centred on the peak of the arc. In many cases it is possible to read the precipitate arcs in the wet gel. We have, however, found it better to $r$ serve final judgment until saline washing (24 h), drying and staining the gel in amido black are completed. Weak precipitates can be seen clearly after this procedure. As the position of the arcs shifts progressively towards the cathode with age of the stain components, stains of a similar age and of known haptoglobin types should be run as controls at the same time as the suspect material. This should be done on each run as slight variation of movement can occur from one run to the next depending on the operator.

Stains up to 3 months old have been correctly typed. The type of cloth which forms the substrate of the stains seems to have only a quantitative effect; more material is required (in area of cloth) if the substrato is cotton than if it is wool. The intensity of the bloodstaining also has considerable bearing on this, of course. Experience is required in estimating the correct amount of material to use.

Many hundreds of those determinations have now been made in this Laboratory both on test and case material and we are satisfied that this is a method which can be used in the routine examination of bloodstains in forensic laboratories.

All this work has been performed with Shandon immunoeloctrophoresis equipment and Dutch Red Cross antisera.

After this work was completed a report by Hallermann, Kiwi and Sturner ${ }^{9}$ came to our notice which describes work on similar lines. Hallermann et al. suggested the use of a modified gel buffer containing glycerine. Its composition is: Solution $A, 7 \cdot 505 \mathrm{~g}$ glycine, $3.85 \mathrm{~g}$ sodium chloride, $1 \mathrm{I}$. water. Solution $B, 0 \cdot 1 \mathrm{M}$ sodium hydroxide. $62.5 \mathrm{ml}$. of solution $A$ are mixed with $37.5 \mathrm{ml}$. of solution $B$ giving a buffer of $p H 10 \cdot 0, \mu=0 \cdot 15$. This is diluted to $\mu=0.05$ and mixed with an equal volume of barbituric lactate gel buffer mentioned earlier. The tank buffer remains the same as before. We have tried this and find that on some occasions the separation is marginally better, while on other occasions no difference can be detected.

Bryan J. CUlliford

B. G. D. WRAXALI

Metropolitan Police Laboratory,

2 Richbell Place,

London, W.C.1.

1 Smithies, O., Biochem. J., 61, 629 (1955)

2 Smithies, O., Nature, 175, 307 (1955).

${ }^{8}$ Smithics, O., and Walker, M. F., Nxture, 176, 1265 (1955).

4 Poulik, M. D., Nature, 180, 1477 (1957).

${ }^{5}$ Culliford, J3. J., Nature, 198, 796 (1963).

- Gervais, P., and Viescou, Cl., Ann. Med. Leg. (1965).

7 Migauchi, C., Rep. Nat. Res. Inst. Police Sci., Tokyo, 17, 3 (1964).

${ }^{8}$ Hirschfeld, J., Sci. Tools, 7, 2 (1960).

- Hallermann, W., Kiwi, M., and Sturner, K. H., Dtsch. Z. ges. gerichtl. Med. 54 (1963).

\section{Morphological Variations in the Eosinophil Granule revealed by Cytochemical Methods}

Morphological variations in the human eosinophil granule under electron microscopic examination have been recently recorded ${ }^{1}$. Furthermore, other variations in the eosinophil granule have been described before ${ }^{1}$. In fact, it seems probable that there are two or more different populations of granules.

This concept is substantiated by my own findings ${ }^{2,3}$. The regularity and morphological features of sudanophilia and peroxidase activity in human peripheral blood eosinophils were studied in an attempt to provide normal data for succeeding investigations. Material was obtained from
104 normal adults of both sexes. The reactions of eosinophils have been observed using Sheehan and Storey's ${ }^{4}$ and Washburn's ${ }^{5}$ methods, for sudanophilia and peroxidase activity, respectively.

Both sudanophilia and peroxidase activity were similar in appearance and intensity in all the granules of 83 per cent $(82.8$ per cent, sudanophilia, 83.1 per cent, peroxidase activity) of the eosinophils. However, in 17 per cent the positive granules showed wide variations in number, size, shape, colour, and distribution. In many cases several varieties of granules occurred in one eosinophil.

Thus, sudanophilia parallels peroxidase activity in human peripheral blood eosinophils and both may exhibit different features in the granules of one eosinophil.

Sufficient material now exists to support a working hypothesis on identification and characterization of distinct eosinophil granules with evolutionary and functional criteria.

\section{Aliberto J. L. MaCario}

Instituto de Investigaciones Hematológicas, Academia Nacional de Medicina, Buenos Aires, Argentina.

1 Ghadially, F. M., and Parry, E. W., Nature, 206, 632 (1965). Ifacario, A. J. L., Rev. Asoc. Bioq. Argentina, 30, 123 (1965).

- ULacario, A. J. L., Rev. Brasil. Med., 22, 730 (1965).

- Sheehan, H. L., and Storey, G. W., J. Path. Bact., 59, 336 (1947). 5 Washburn, A. H., J. Lab. Clin. Med., 14, 246 (1928).

\section{Coagulant Activity of Tiger Snake (Notechis scutatus scutatus) Venom}

TrGer snake venom has the ability to clot blood. Holden ${ }^{1}$ and Blömback ${ }^{2}$ have presented evidence that prothrombin, Factor $\mathrm{V}$ and calcium ions are required for the venom to convert fibrinogen to fibrin. We have studied the action of this venom on several purified clotting factors. As the crude venom was used in these experiments, the results obtained may arise from more than one active constituent present in the venom.

In preliminary experiments with plasma systems, it was confirmed that the venom exerts its optimal coagulant activity only in the presence of prothrombin, Factor V, a divalent metal (calcium, barium, strontium or manganese), and a phospholipid emulsion which is able to increase the rate of activation of prothrombin. The venom had very little coagulant activity on purified human fibrinogen (Kabi Pharmaceuticals, Ltd.). Furthermore, the venom neither activated nor destroyed Factor $X$. When purified bovine Factor $\mathrm{X}$ (ref. 3), or Factor $\mathrm{X}$ in human serum, was incubated at $37^{\circ} \mathrm{C}$ with tiger snake venom (KochLight Laboratories, Ltd.), phospholipid and calcium ions, no activated Factor $\mathrm{X}$ was produced; if, however, after 30 min Russell's viper venom was added, it converted all the Factor $\mathrm{X}$ to the active form $(\mathrm{X} a)$. This is interesting in itself, as no other venom has yet been shown to be capable of activating prothrombin and incapable of activating Factor $\mathrm{X}$ (refs. 4 and 5).

Blömback ${ }^{2}$ has proposed that the coagulant substance of tiger snake venom acts on Factor $V$, and as a result Factor $\mathrm{V}$ acquires the ability to activate prothrombin. In his experiments, incubation of a partially purified Factor V preparation with the venom produced a prothrombin converting activity which increased with time. However, we have repeated these experiments with pure bovine Factor V (ref. 6), and have found that incubation of Factor V with the venom, with or without phospholipid ('Inosithin') and ealcium ions, produced no progressive increase in coagulant activity, but rather a gradual inactivation occurred.

The rate of activation of purified bovine prothrombin by the venom in purified systems was studied, with conditions as described elsewhere ${ }^{7}$. The effect of adding Factor V, phospholipid (phosphatidic acid/phosphatidylcholine, $1: 1$ ) and calcium ions to prothrombin and the 